\title{
Impact and sustainability of centralising acute stroke services in English metropolitan areas: retrospective analysis of hospital episode statistics and stroke national audit data
}

\author{
Stephen Morris, ${ }^{1}$ Angus I G Ramsay, ${ }^{1}$ Ruth J Boaden, ${ }^{2}$ Rachael M Hunter, ${ }^{3}$ Christopher McKevitt, \\ Lizz Paley, ${ }^{5}$ Catherine Perry, ${ }^{2}$ Anthony G Rudd, ${ }^{6}$ Simon J Turner, ${ }^{7}$ Pippa J Tyrrell, ${ }^{8}$ \\ Charles D A Wolfe, ${ }^{4,9}$ Naomi J Fulop ${ }^{1}$
}

For numbered affiliations see end of article.

Correspondence to: S Morris steve.morris@ucl.ac.uk (ORCID 0000-0002-5828-3563)

Additional material is published online only. To view please visit the journal online.

Cite this as: BMJ 2019;364:11 http://dx.doi.org/10.1136/bmj.l1

Accepted: 20 December 2018

\section{ABSTRACT}

OBJECTIVES

To investigate whether further centralisation of acute stroke services in Greater Manchester in 2015 was associated with changes in outcomes and whether the effects of centralisation of acute stroke services in London in 2010 were sustained.

\section{DESIGN}

Retrospective analyses of patient level data from the Hospital Episode Statistics (HES) database linked to mortality data from the Office for National Statistics, and the Sentinel Stroke National Audit Programme (SSNAP).

SETTING

Acute stroke services in Greater Manchester and London, England.

\section{PARTICIPANTS}

509182 stroke patients in HES living in urban areas admitted between January 2008 and March 2016; 218120 stroke patients in SSNAP between April 2013 and March 2016.

INTERVENTIONS

Hub and spoke models for acute stroke care.

MAIN OUTCOME MEASURES

Mortality at 90 days after hospital admission; length of acute hospital stay; treatment in a hyperacute stroke unit; 19 evidence based clinical interventions.

\section{WHAT IS ALREADY KNOWN ON THIS TOPIC}

In 2010 acute stroke services were centralised across two metropolitan areas of England

In London, where the policy was to provide hyperacute stroke care to all patients, a reduction in mortality and length of hospital stay was seen

In Greater Manchester, where hyperacute stroke care was provided only to patients presenting within four hours of developing symptoms, no impact on mortality was seen but length of hospital stay decreased

Further centralisation of the acute stroke system in Greater Manchester took place in 2015, whereby all stroke patients were eligible for treatment in a hyperacute stroke unit

\section{WHAT THIS STUDY ADDS}

After further centralisation in Greater Manchester, a significant decline in mortality occurred among patients treated at a hyperacute stroke unit and overall length of acute hospital stay fell

Effects of centralisation in London on mortality and length of hospital stay were maintained from 2010 to 2016

This study provides further evidence for centralising acute stroke services in hub and spoke models in urban areas whereby all stroke patients receive hyperacute stroke care

\section{RESULTS}

In Greater Manchester, borderline evidence suggested that risk adjusted mortality at 90 days declined overall; a significant decline in mortality was seen among patients treated at a hyperacute stroke unit (difference-in-differences -1.8\% (95\% confidence interval -3.4 to -0.2$)$ ), indicating 69 fewer deaths per year. A significant decline was seen in risk adjusted length of acute hospital stay overall $(-1.5(-2.5$ to -0.4) days; P<0.01), indicating 6750 fewer bed days a year. The number of patients treated in a hyperacute stroke unit increased from $39 \%$ in $2010-12$ to $86 \%$ in 2015/16. In London, the 90 day mortality rate was sustained ( $P>0.05)$, length of hospital stay declined ( $P<0.01$ ), and more than $90 \%$ of patients were treated in a hyperacute stroke unit. Achievement of evidence based clinical interventions generally remained constant or improved in both areas.

\section{CONCLUSIONS}

Centralised models of acute stroke care, in which all stroke patients receive hyperacute care, can reduce mortality and length of acute hospital stay and improve provision of evidence based clinical interventions. Effects can be sustained over time.

\section{Introduction}

Stroke is a leading cause of mortality and disability. ${ }^{1}$ A wealth of evidence shows that organised care in an inpatient stroke unit is associated with better quality of care and reduced death and dependency. ${ }^{2-4}$ Evidence also suggests that variation exists within and between countries in both access to stroke unit care and organisational models within stroke units, ${ }^{56}$ which affects access to rapid diagnosis and treatment, secondary prevention, and rehabilitation. ${ }^{7}$ In several countries, acute stroke services are being centralised into "hub and spoke" systems as a means of improving access to organised care in inpatient stroke units. Hospitals providing different levels of stroke care work together to create a centralised system, ${ }^{8}$ in which all patients in the system with acute stroke are taken to central specialist units rather than the nearest hospital, with a larger number of hospitals providing care beyond the acute phase. Research in several countries including the US, Canada, the Netherlands, Denmark, and Australia suggests that this approach may improve provision of evidence based care processes for all stroke patients, by increasing access to specialist care and thrombolysis where appropriate. ${ }^{9-14}$ Although improved clinical outcomes 
among patients admitted to organised inpatient stroke care are well documented, relatively little evidence exists to show whether centralising acute stroke care to a small number of high volume specialist centres produces better clinical outcomes at the system level across all stroke patients. ${ }^{15} 16$ Our previous research showed reductions in mortality after the centralisation of stroke services in London (see below). A recent study found that centralisation in the Central Denmark Region significantly reduced length of acute hospital stay without compromising quality, and mortality and readmissions remained unchanged. ${ }^{17}$ No studies have evaluated longer term sustainability of the effects of centralising acute stroke services.

In 2010 acute stroke services were centralised across two metropolitan areas of England-Greater Manchester (population 2.7 million) and London (8.2 million).$^{18}$ Our earlier analysis using data from January 2008 to March 2012 showed that in London, where hyperacute stroke care was provided to all patients, a reduction in mortality and length of acute hospital stay occurred; in Greater Manchester, where specialist hyperacute stroke care was provided only to patients presenting within four hours of developing stroke symptoms, no effect on mortality was seen but length of hospital stay decreased. ${ }^{19}$ We also showed that patients treated at hyperacute stroke units (HASUs) in Greater Manchester and London were significantly more likely to receive evidence based interventions than those treated at other units. ${ }^{20}$ In Greater Manchester, 39\% of stroke patients were admitted to a HASU, compared with $93 \%$ in London, possibly explaining the difference in mortality outcomes between the two areas. Both centralisations had a high probability of being cost effective. ${ }^{21}$ Other research has shown that outcomes for patients treated at HASUs in London improved after the centralisation, whereas outcomes worsened for those in London not treated at HASUs ${ }^{22}$; differences in in-hospital mortality between weekday and weekend admissions fell in London between 2008 and 2014, but this was not related to the centralisation ${ }^{23}$; and the centralisation in London was cost effective. ${ }^{24}$

In Greater Manchester, further centralisation of the acute stroke system was implemented in April 2015; also, few data are available on the sustainability of the effects of centralising acute stroke services. Given this, plus the growing interest in reconfiguration of services generally, ${ }^{25}$ we extended our original analyses with new data covering the period to March 2016. The aims of this study were to investigate whether the further centralisation of acute stroke services in Greater Manchester in 2015 had an effect on outcomes and whether the effects of the 2010 centralisation in London were sustained over an additional four years (2012-16). We also analysed provision of evidence based interventions, as these may explain outcomes. ${ }^{20}$

\section{Methods}

The centralisations

Before the centralisations, in both London and Greater Manchester, patients with suspected stroke were taken to the nearest emergency department to receive stroke care and then treated on a stroke unit or general ward (fig 1, first panel). After the centralisation in London in 2010, 24/7 specialist care was provided to all patients in eight designated HASUs. All patients with suspected stroke (having a deficit on one or more of the face, arm, and speech elements of the FAST test) were taken by the London Ambulance Service to the HASU that involved the shortest journey time (no more than 30 minutes away). The appropriate HASU for a particular postcode may change depending on traffic conditions. Ambulance staff may not necessarily transport patients to the nearest HASU depending on capacity issues. On arrival at the HASU, patients are assessed immediately by specialised stroke medical teams capable of rapid brain imaging and thrombolysis when appropriate. Patients are then admitted to a HASU bed where they receive hyperacute care for up to 72 hours after admission. Once patients are stabilised, they are either transferred to one of 24 stroke units that provide acute rehabilitation services and ongoing medical supervision or discharged home and receive community rehabilitation services (fig 1, second panel). ${ }^{18}$ The location of the stroke unit that patients are taken to depends on their postcode of residence; patients are usually transferred to a stroke unit close to their home, which may be in the same hospital as the HASU. Length of stay in the stroke unit varies, lasting until the patient is well enough to be discharged from an acute inpatient setting. Following their stay in the stroke unit, patients are discharged home or to a specialist inpatient facility for long term rehabilitation. After the 2010 reconfiguration in Greater Manchester (until 2015), patients presenting within four hours of developing stroke symptoms were taken directly to a HASU (one 24/7 HASU called a comprehensive stroke centre (CSC) and two HASUs running $7 \mathrm{am}-7 \mathrm{pm}$, Monday-Friday (primary stroke centres; PSCs)); all other patients were taken to one of 10 district stroke centres (DSCs), designated to provide all aspects of post-thrombolysis stroke care (fig 1 , third panel). ${ }^{26}$ After the 2015 centralisation in Greater Manchester, all patients were eligible for treatment in a HASU rather than just those arriving at hospital within four hours, and the two HASUs operating "in hours" extended the hours in which they would admit stroke patients (7am-11pm, Monday-Sunday) (fig 1, fourth panel). The HASUs in Greater Manchester operate similarly to those in London.

\section{Data}

Analysis of mortality and length of acute hospital stay

To investigate mortality and length of acute hospital stay, we obtained patient level data from the Hospital Episode Statistics (HES) database for all patients in England with a primary diagnosis of stroke defined using international classification of diseases, 10th revision (ICD-10) codes I61 (intracerebral haemorrhage), I63 (cerebral infarction), or I64 (stroke, not specified as haemorrhage or infarction, which usually refers to 

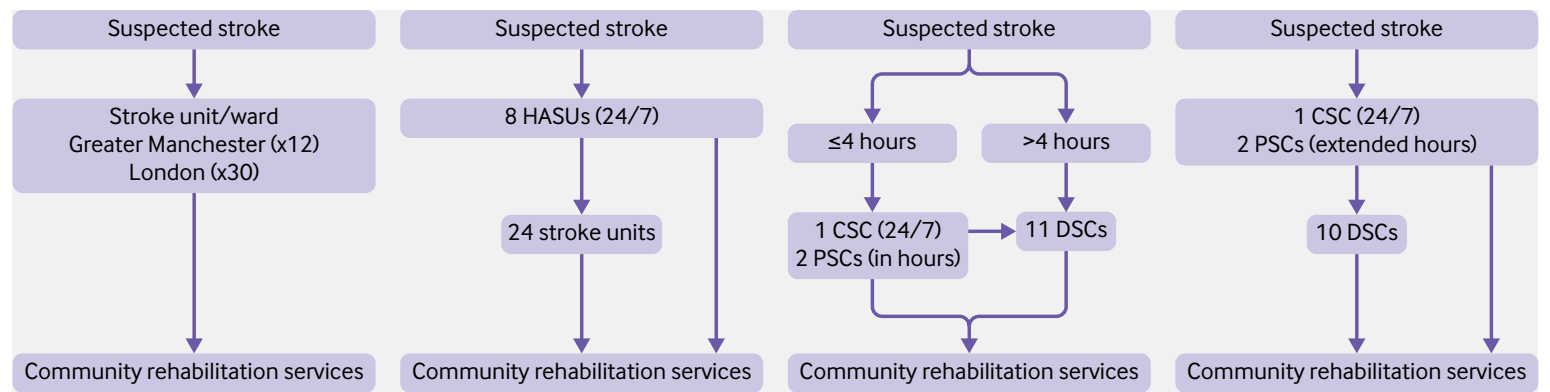

Fig 1 | Simplified pre-centralisation and post-centralisation models in London and Greater Manchester. First panel: before 2010 centralisations in London and Greater Manchester. Second panel: after 2010 centralisation in London. Third panel: after 2010 centralisation in Greater Manchester until 2015. Fourth panel: after 2015 centralisation in Greater Manchester. CSC=comprehensive stroke centre; $D S C=$ district stroke centre; $\mathrm{HASU}=$ hyperacute stroke unit; $\mathrm{PSC}=$ primary stroke centre.

cases in which the cause of the stroke has not been documented in the medical record) between 1 January 2008 and 31 March 2016 (that is, 33 quarters/99 months). ${ }^{27}$ We excluded subarachnoid haemorrhage (ICD-10 code I60) as it is managed through a different clinical pathway. ${ }^{28}$ The dataset was confined to patients living in urban areas (defined as "urban-less sparse" using the urban/rural classification for England). ${ }^{29} \mathrm{We}$ linked the data to mortality data supplied by the Office for National Statistics, ${ }^{30}$ using an anonymised unique patient identifier to identify deaths from any cause and at any place of death (hospital or otherwise) by 90 days after hospital admission. We measured length of acute hospital stay (HASU plus stroke unit stay) in days as the difference between date of admission and date of discharge, including same day transfers between hospitals. We created regional variables to identify whether the hospital trust the patient was admitted to was in London, Greater Manchester, or the rest of England. The start of our analysis period was January 2008, following the publication of the National Stroke Strategy for the English NHS in December 2007. ${ }^{31}$ The centralisations in Greater Manchester were completed in April 2010 and March/April 2015. The centralisation in London began to be implemented in October 2009 and was fully operational in July 2010. Data were available for 509182 admissions, of which 34051 were in Greater Manchester (9412 from January 2008 to March 2010, 20390 from April 2010 to March 2015, 4249 from April 2015 to March 2016), 69847 were in London (18654 before the centralisation, 51193 after), and 405284 were for the rest of England. Mortality data for our analysis were complete; we dropped 17411 (3\%) observations from the analysis of length of acute hospital stay because admission or discharge data were missing (4207 observations) or the acute hospital stay lasted more than 120 days (13 204).

\section{Analysis of evidence based clinical interventions}

To investigate evidence based clinical interventions, we used patient level data collected for the clinical audit component of the Sentinel Stroke National Audit Programme (SSNAP), ${ }^{32}$ organised by the Royal College of Physicians. Our data covered the period 1 April 2013 to 31 March 2016 and included data submitted by every stroke unit in England. We examined whether the patient was treated in a HASU, plus 19 binary clinical interventions (measured from time of hospital arrival or onset of stroke symptoms for patients who were already in hospital): brain scan within 60 minutes, 180 minutes, or 24 hours; administration of intravenous thrombolysis (tissue plasminogen activator) if eligible, and whether it was given within 60 minutes; swallow screen within four hours; admission to a stroke unit within four hours; assessment by a stroke consultant physician within 14 hours or 24 hours; assessment by a stroke nurse within 12 hours or 24 hours; assessment by a specialist physiotherapist within 24 hours or 72 hours; assessment by an occupational therapist within 24 hours or 72 hours; formal swallow assessment by a speech and language therapist within 24 hours or 72 hours; and communication assessment by a speech and language therapist within 24 hours or 72 hours. These measures are quality interventions routinely reported by SSNAP. Data were available for 218120 admissions, of which 12086 were in Greater Manchester (7606 from April 2013 to March 2015, 4480 from April 2015 to March 2016), 24281 were in London, and 181753 were for the rest of England.

\section{Statistical analyses}

We evaluated whether the 2015 centralisation of acute stroke services in Greater Manchester had an effect on mortality and length of hospital stay by using a between region difference-in-differences regression analysis comparing the change over time in Greater Manchester with the change over time in the rest of England (controlling for outcomes in London). We did the analysis at hospital level, using monthly observations of risk adjusted mortality and length of hospital stay. The risk adjustment was done at the patient level. We calculated expected risks of death at 90 days after admission by using patient level logistic regressions, including binary indicators for sex and age interactions (age measured in five year bands), stroke diagnosis using the first four digits of the primary ICD-10 diagnostic code (19 categories), number of comorbidities derived from secondary ICD10 diagnostic codes, presence of 16 comorbidities based on ICD-10 chapter headings derived from secondary ICD-10 diagnostic codes, ethnic group (19 categories), and fifth of deprivation of the lower 
layer super output areas in which the patient lived. ${ }^{33}$ We ran the patient level regressions only for patients who had a stroke before the centralisations so that risk adjustment was not contaminated by the changes. We used the regression coefficients (derived from the logistic regressions for the pre-implementation period) to predict the probability of mortality for every patient (in both pre-implementation and post-implementation periods). We aggregated these to create a dataset of the actual percentage of patients who died and the expected percentage by admitting hospital and month. We tested whether the 2015 centralisation had an effect on mortality by using least squares regression of the actual minus expected mortality percentage against interaction terms between Greater Manchester and the post-April 2015 centralisation period. We included binary indicators for admitting hospital (520 hospital fixed effects) and month (99 time fixed effects), as well as an interaction term between London and the post-July 2010 London centralisation period to control for the effects of the London reconfiguration. We used a similar method in our previous study, ${ }^{19}$ and before that it was used in an evaluation of the advancing quality initiative in the north west of England. ${ }^{34}$ Some hospitals began to reconfigure their services before April 2015, and we controlled for this in our analysis by using hospital and time fixed effects. We did not include a separate interaction term for the April 2010 centralisation in Greater Manchester because our previous analysis showed no effect of this reconfiguration on mortality. Each observation was weighted by the number of patients treated at that hospital in that month. We re-ran the second stage of the difference-in-differences analysis including an interaction term only for the HASUs and the post-centralisation period (note that the primary stroke centres were not 24/7 HASUs, but data on time of arrival at the hospital were not available so the analysis included all patients treated at these hospitals irrespective of when they arrived). We ran all of the above separately for stroke diagnoses (ICD-10 codes I61, I63, and I64).

We used the same approach for length of hospital stay in Greater Manchester, but our risk adjustment equation was estimated using a generalised linear model with $\gamma$ family and log link to account for data skewness. ${ }^{35}$ We also added binary indicators for mortality at three, 30, and 90 days after stroke to the risk equation and used the regression coefficients to predict expected mean length of acute hospital stay. We included a separate interaction term for the pre-April 2010 centralisation in Greater Manchester because our previous analysis showed a significant effect of the 2010 centralisation on length of hospital stay; our comparison was therefore of the change in length of stay following the second centralisation compared with the first.

To analyse whether the effects of the 2010 centralisation in London were sustained over the period 2012-16, using patient level data for London only we regressed 90 day mortality against the covariates used in the risk adjustment model described above, plus hospital and month. We used logistic regression analysis. We then calculated the predictive margins of month on mortality and plotted these on a graph. We tested for significant variations in adjusted mortality since the centralisation by using Wald tests, and we present the results as $\mathrm{P}$ values under the null hypothesis that the regression coefficient for every month after the centralisation (which was fully operational from July 2010) was the same as the regression coefficient for July 2010. In the analysis of length of hospital stay in London, we used a similar approach except that we used the generalised linear model as above and also controlled for mortality at three, 30, and 90 days.

For the analysis of the evidence based clinical interventions, we used all data submitted across England and regressed whether or not each of the clinical interventions had been achieved against sex, age (measured in five year bands), stroke diagnosis (cerebral infarction or intracerebral haemorrhage), whether the stroke happened inside or outside hospital, and worst level of consciousness on arrival at the hospital (alert, verbally arousable, not alert, or totally unresponsive). ${ }^{36}$ We used logistic regression analysis and included interactions terms between time period (1 April 2013 to 31 March 2014, 1 April 2014 to 31 March 2015, 1 April 2015 to 31 March 2016) and region (Greater Manchester, London, rest of England), and we calculated predictive margins for each of these interaction terms to give the adjusted probability that each clinical intervention would be achieved in each region and year, controlling for the covariates. We did not include hospital fixed effects as these were perfectly correlated with region. In Greater Manchester, we were interested in whether achievement of the clinical interventions improved in the third year of our data beyond the changes seen in the rest of England. In London, we were interested in whether the achievement of the clinical interventions was constant over the three year period. For comparison, we present the findings alongside the results of our original analyses covering the period 2010-12, which included fewer clinical interventions. ${ }^{20}$

\section{Patient and public involvement}

This study was supported by a patient and families research advisory group that was consulted on the original research question and methods for disseminating the outputs of this study. We also presented findings from previous analyses done as part of this study to these groups to ensure that we were addressing questions and communicating lessons in a meaningful way. The findings of this research will be disseminated to the relevant patient community in an accessible way.

\section{Results}

\section{Outcomes}

Across all stroke types combined, unadjusted mortality at 90 days in Greater Manchester fell by 3.1 percentage 
points after the April 2015 centralisation; in the rest of England, the reduction was $2.0 \%$ (table 1). The unadjusted between region difference-in-differences indicate an additional $1.1 \%$ reduction in 90 day mortality in Greater Manchester over and above the reduction seen in the rest of England.

We found borderline evidence of adjusted between region difference-in-differences in 90 day mortality in Greater Manchester across all types of stroke combined (95\% confidence interval -2.7 to 0.01 ; table 2 ). When we re-ran the analysis only for patients treated at a HASU ( $86 \%$ of all stroke patients, see below), we saw a $1.8 \%(-3.4$ to -0.2$)$ reduction in 90 day mortality (table $2)$; the coefficient was larger (more negative) than in the all hospital model and significantly different from zero. Assuming an estimated 4500 strokes in Greater Manchester each year (see above), and that $86 \%$ of these were treated in a HASU (see below), the mortality reduction is consistent with 69 fewer deaths per year $(4500 \times 0.86 \times-1.8 / 100)$.

Values in difference-in-differences columns are absolute differences in risk adjusted mortality showing change over time in Greater Manchester minus change over time in rest of England (see text). Values in preceding columns show absolute risk adjusted mortality in rest of England, Greater Manchester, and Greater Manchester HASUs. To estimate these, separate models were run for rest of England, Greater Manchester, and Greater Manchester HASUs. Patient level data were used to regress mortality against sex and age interactions, stroke diagnosis using first four digits of primary ICD-10 diagnostic code, number of comorbidities derived from secondary ICD-10 diagnostic codes, presence of 16 comorbidities based on ICD-10 chapter headings derived from secondary ICD10 diagnostic codes, ethnic group, fifth of deprivation, hospital and calendar month, plus indicator variables for whether patient was admitted before or after April 2015 reconfiguration in Greater Manchester; predictive margins for this indicator variable are shown in table. Values in difference-in-differences columns do not equal differences in absolute risk adjusted mortality in preceding columns: with statistical method used to estimate difference-in-differences (see text), it is not possible to recover absolute risk adjusted mortality levels directly.

In Greater Manchester, mean unadjusted length of acute hospital stay across all stroke types was 17.4 days between April 2010 and March 2015 and 14.4 days after the 2015 centralisation; figures in the rest of England were 18.1 days and 15.7 days, respectively (table 3). The unadjusted between region differencein-differences in length of hospital stay was -0.6 days.

Across all stroke types, we found a significantly larger decline in risk adjusted length of acute hospital stay in Greater Manchester compared with the rest of England, by 1.5 ( -2.5 to -0.4 ) days (table 4). If this reduction was applied to the estimated 4500 patients with a stroke in Greater Manchester each year, this would result in 6750 fewer bed days a year.

We re-ran our models on patients stratified by type of stroke (see supplementary table A for further details of the numbers of patients with each type of stroke in each time period and region), and found that reductions in mortality and length of hospital stay were largely achieved among patients diagnosed as having cerebral infarction (table 2 and table 4), who were the majority of cases (supplementary table A). For length of acute hospital stay, the largest decline was in the I64 (stroke unspecified) subgroup, which may be a function of better access to diagnostic testing on arrival at the hospital (especially in patients admitted to a HASU), and, as a consequence of this, declining numbers of patients falling into this group (supplementary table A). Mortality and length of stay outcomes for patients with intracerebral haemorrhage were non-significant, possibly owing to the relatively small numbers of patients in the subgroup (supplementary table A).

In London, we found no significant variation in mortality at 90 days over time since the centralisation ( $\mathrm{P}=0.09$; fig 2). Length of acute hospital stay since the centralisation significantly declined $(\mathrm{P}<0.01$; fig 3$)$.

\section{Treatment in hyperacute stroke unit}

In Greater Manchester, the percentage of patients treated in a HASU increased from $39 \%$ in $2010-12$ to $57 \%$ in $2013 / 14$, to $64 \%$ in $2014 / 15$, and to $86 \%$ in 2015/16 (supplementary table B). Equivalent figures for patients in London treated in a HASU were 93\%, $95 \%, 94 \%$, and $94 \%$, respectively.

\section{Evidence based clinical interventions}

In Greater Manchester, achievement of evidence based clinical interventions after the 2015 centralisation either stayed the same or improved compared with the previous two years (table 5). Improvements of

\begin{tabular}{|c|c|c|}
\hline Analysis & No of admissions & Mortality (\%) \\
\hline Rest of England (April 2015 to March 2016) & 48443 & 21.3 \\
\hline Greater Manchester (January 2008 to March 2015) & 29802 & 22.7 \\
\hline Greater Manchester (April 2015 to March 2016) & 4249 & 19.6 \\
\hline Rest of England (April 2015 to March 2016) minus (January 2008 to March 2015) & - & -2.0 \\
\hline Greater Manchester (April 2015 to March 2016) minus (January 2008 to March 2015) & - & -3.1 \\
\hline \multicolumn{3}{|l|}{ Difference-in-differences: } \\
\hline Greater Manchester minus rest of England & - & -1.1 \\
\hline
\end{tabular}




\begin{tabular}{|c|c|c|c|c|c|c|c|c|}
\hline \multirow[b]{2}{*}{ Type of stroke } & \multicolumn{2}{|c|}{ Rest of England } & \multicolumn{2}{|c|}{ Greater Manchester } & \multicolumn{2}{|c|}{ HASUs in Greater Manchester } & \multicolumn{2}{|c|}{ Difference-in-differences $(95 \% \mathrm{Cl})$} \\
\hline & Before & After & Before & After & Before & After & $\begin{array}{l}\text { Greater Manchester v } \\
\text { rest of England }\end{array}$ & $\begin{array}{l}\text { HASUs in Greater Manchester v } \\
\text { rest of England }\end{array}$ \\
\hline All stroke types & 23.4 & 20.8 & 22.8 & 18.6 & 23.5 & 18.4 & $-1.3(-2.7$ to 0.01$)$ & $-1.8(-3.4$ to -0.2$)$ \\
\hline Intracerebral haemorrhage $\left(161^{\star}\right)$ & 41.9 & 43.4 & 42.6 & 39.7 & 43.1 & 39.9 & $-4.1(-8.4$ to 0.2$)$ & $-5.0(-10.3$ to 0.4$)$ \\
\hline Cerebral infarction $\left(163^{\star}\right)$ & 19.9 & 17.0 & 19.8 & 15.8 & 20.5 & 15.4 & $-1.2(-2.6$ to 0.2$)$ & $-1.9(-3.6$ to -0.3$)$ \\
\hline $\begin{array}{l}\text { Stroke, not specified as } \\
\text { haemorrhage or infarction }\left(164^{\star}\right)\end{array}$ & 24.4 & 19.3 & 21.6 & 16.3 & 22.7 & 19.7 & $-1.0(-5.8$ to 3.7$)$ & $4.6(-3.8$ to 12.9$)$ \\
\hline
\end{tabular}

greater than $10 \%$ occurred for several "front door" processes (brain scan within 60 minutes, admitted to a stroke unit within four hours) and assessments over the first 72 hours (assessment by a stroke consultant physician within 14 and 24 hours, assessment by a specialist physiotherapist with 24 hours, assessment by an occupational therapist within 24 hours, and communication assessment by a speech and language therapist within 24 hours). Improvements and levels of achievement in the rest of England during the same time period were either similar to or lower than those seen in Greater Manchester. In London the "front door" processes, use of thrombolysis, and assessments during the first 72 hours all stayed high and constant over the three years with no more than a 5\% fluctuation each year (table 6). The percentage of patients admitted to a stroke unit within four hours and the percentage having a formal swallow assessment by a speech and language therapist within 72 hours fell by $5-10 \%$. We identified similar trends when we used unadjusted data (supplementary tables C-E).

\section{Discussion}

After the further centralisation of stroke care in Greater Manchester in 2015, a larger proportion of patients were treated in a HASU (comprehensive stroke centre or primary stroke centre) than in 2010. Achievement of evidence based clinical interventions either stayed the same or improved compared with the rest of England. A decline in mortality from stroke occurred after the centralisation, and we found borderline evidence that

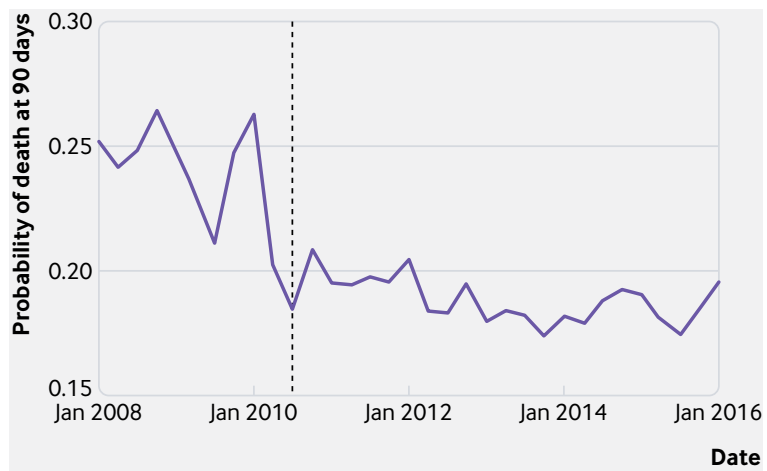

Fig 2 | Adjusted trends in mortality at 90 days in London. Note that y axis does not start at zero. Vertical line indicates when centralisation in London was fully operational (July 2010), although centralisation began to be implemented in October 2009. P value (under null hypothesis that regression coefficient for every month after centralisation (which occurred in July 2010) is same as regression coefficient for July 2010) is 0.09 a reduction in mortality occurred across all hospitals in Greater Manchester over and above the changes seen in the rest of England. A reduction in all cause mortality was seen in the HASUs that was statistically significantly larger than the reductions seen elsewhere and would amount to approximately 69 fewer deaths a year. A significant reduction in length of hospital stay of 1.5 days per patient was seen in Greater Manchester over and above the reduction seen in the rest of England, which would amount to approximately 6750 fewer bed days a year. In the analysis for London, mortality and evidence based clinical interventions seem to have varied little over time since the centralisation in July 2010. Length of acute hospital stay since the centralisation significantly declined. This indicates that the improvements seen after the centralisation in London have largely been sustained, with reductions in performance in some clinical interventions and further declines in length of acute hospital stay.

\section{Strengths and weaknesses of study}

The strengths and weakness are similar to those of our earlier studies. ${ }^{1920}$ The strengths relate to the large national datasets and the robust quasi-experimental analytical frameworks that we used. In addition, our data covered a longer time period allowing us to investigate the effect of further changes in Greater Manchester and whether the improvements in London seen in 2010 to 2012 were maintained over time.

The main limitation is lack of data on severity of stroke; these data are not available in the HES database. If stroke patients were more likely to die before reaching the HASU owing to longer travel distances, or if more patients with less severe strokes were likely to go to hospital after centralisation, this would mean that the level of stroke severity on arrival at the hospital would be different before and after centralisation. As shown in the supplementary material, no discernible differences existed in indicators of stroke severity (worst level of consciousness in first 24 hours after stroke) over time in patients admitted to hospital in Greater Manchester and London. Nevertheless, we cannot rule out the possibility that our findings are due to variations in stroke severity between Greater Manchester, the rest of England, and London over time.

Another limitation is that we were unable to include other important outcomes such as disability. Data on disability and dependence are collected in SSNAP at six month follow-up assessments but are not collected 


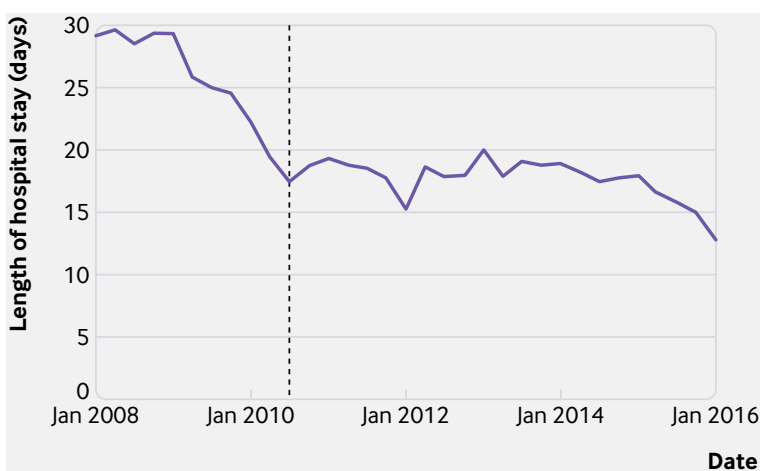

Fig 3 | Adjusted trends in length of hospital stay in London. Vertical line indicates when centralisation in London was fully operational (July 2010), although centralisation began to be implemented in October 2009. P value (under null hypothesis that regression coefficient for every month after centralisation (which occurred in July 2010) is same as regression coefficient for July 2010) is $\mathbf{2 0 . 0 1 \text { ) }}$

reliably enough for analysis. Additional limitations include lack of data on quality of life, lack of data on pre-hospital processes and outcomes, and limitations in measurement of length of hospital stay when patients may be repatriated between stroke units. Additionally, length of acute hospital stay depends on the availability of community rehabilitation services and the local agreements between acute and rehabilitation services on the timing of transmission of stroke patients from acute care to rehabilitation. Although we controlled for hospital and time specific effects in our analysis, the decrease in length of acute hospital stay may be explained by arrangements for earlier transmission of patients to rehabilitation units from acute stroke services. In addition, the primary stroke centres in Greater Manchester were not 24/7 hyperacute stroke units, but data on time of arrival at the hospital were not available so the analysis included all patients treated at these hospitals irrespective of when they arrived (that is, we included people who were treated at the primary stroke centres even when they were not operating as HASUs), which may mean we have underestimated the impact of centralisation in Greater Manchester. A limitation of our analysis of SSNAP data is that achievement of clinical interventions was measured from time of arrival at hospital rather than from onset of symptoms. Finally, our analysis used data only for patients treated up to one year after the further centralisation in Greater Manchester.

Strengths and weaknesses in relation to other studies Our findings extend those of our earlier analyses, examining the longer term effects of the centralisation in London, whether the further centralisation in Greater Manchester was associated with improvements in outcomes, and achievement of clinical interventions. The improvement in outcomes seen in London has been borne out in other studies, although these have also shown that outcomes worsened for patients treated for acute stroke in London but not in a HASU. ${ }^{22}$ Our findings are consistent with a previous analysis based on national stroke audit data showing that patients admitted to stroke services with higher levels of organisation were more likely to receive high quality care and to have a reduced risk of death 30 days after stroke. ${ }^{2}$ Our results are also consistent with those of a study evaluating the centralisation of acute stroke services in Denmark, which used national stroke registry data to show that centralisation reduced length of hospital stay without compromising quality of care. ${ }^{17}$ These studies are important because both were able to control for stroke severity. Our study has focused on one model of acute stroke care-centralised "hub and spoke" systems-but other models are also possible. For example, evidence from a systematic review suggests that for patients with ischaemic stroke the location of initial thrombolysis treatment does not affect outcomes; death and disability rates were not significantly different among thrombolysed patients starting treatment in non-specialist centres versus specialist centres. ${ }^{37}$ The greater travel times in rural areas make centralisation challenging and may necessitate other solutions, such as telemedicine; in Finland, a decentralised telestroke thrombolysis service achieved similar treatment rates and time delays for a rural population to those achieved by a centralised system for an urban population. ${ }^{38}$

\section{Meaning of study}

Our study provides evidence to support the centralisation of acute stroke services in urban areas and shows that this service model should include all stroke patients in those areas. This is supported by our

\begin{tabular}{|c|c|c|}
\hline Analysis & No of admissions & Unadjusted length of hospital stay (mean days) \\
\hline Rest of England (January 2008 to March 2010) & 105456 & 21.0 \\
\hline Rest of England (April 2010 to March 2015) & 240369 & 18.1 \\
\hline Rest of England (April 2015 to March 2016) & 47507 & 15.7 \\
\hline Greater Manchester (January 2008 to March 2010) & 8862 & 21.7 \\
\hline Greater Manchester (April 2010 to March 2015) & 18994 & 17.4 \\
\hline Greater Manchester (April 2015 to March 2016) & 4054 & 14.4 \\
\hline \multicolumn{3}{|l|}{ Differences: } \\
\hline Rest of England (April 2015 to March 2016) minus (April 2010 to March 2015) & - & -2.4 \\
\hline Greater Manchester (April 2015 to March 2016) minus (April 2010 to March 2015) & - & -3.0 \\
\hline \multicolumn{3}{|l|}{ Difference-in-differences: } \\
\hline Greater Manchester minus rest of England & - & -0.6 \\
\hline
\end{tabular}




\begin{tabular}{|c|c|c|c|c|c|}
\hline \multirow[b]{2}{*}{ Type of stroke } & \multicolumn{2}{|c|}{ Rest of England } & \multicolumn{2}{|c|}{ Greater Manchester } & \multirow{2}{*}{$\begin{array}{l}\text { Difference-in-differences }(95 \% \mathrm{Cl}) \text { : Greater Manchester v rest of } \\
\text { England }\end{array}$} \\
\hline & Before & After & Before & After & \\
\hline All stroke subtypes & 19.7 & 15.7 & 19.6 & 13.2 & $-1.5(-2.5$ to -0.4$)$ \\
\hline Intracerebral haemorrhage $\left(161^{\star}\right)$ & 19.8 & 16.9 & 19.7 & 15.9 & $-1.2(-3.3$ to 1.0$)$ \\
\hline Cerebral infarction $\left(163^{*}\right)$ & 20.4 & 16.2 & 21.0 & 13.5 & $-1.3(-2.4$ to -0.2$)$ \\
\hline Stroke, not specified as haemorrhage or infarction $\left(164^{\star}\right)$ & 15.9 & 12.0 & 15.0 & 11.6 & $-4.4(-7.0$ to -1.8$)$ \\
\hline
\end{tabular}

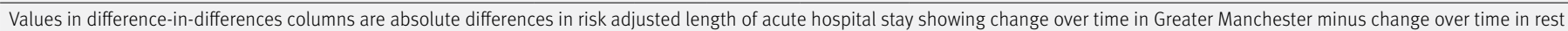
of England (see text). Values in preceding columns show absolute risk adjusted length of hospital stay in rest of England and Greater Manchester. To estimate these, separate models were run for rest of England and Greater Manchester. Patient level data were used to regress length of hospital stay against sex and age interactions, stroke diagnosis using first four digits of primary ICD-10 diagnostic code, number of comorbidities derived from secondary ICD-10 diagnostic codes, presence of 16 comorbidities based on ICD-10 chapter headings derived from secondary ICD-10 diagnostic codes, ethnic group, fifth of deprivation, hospital and calendar month, mortality at 3, 30, and 90 days, plus indicator variables for whether patient was admitted before or after April 2015 reconfiguration in Greater Manchester; predictive margins for this indicator variable are shown in table. Values in difference-in-differences columns do not equal differences in absolute risk adjusted length of hospital stay in preceding columns: with statistical method used to estimate difference-in-differences (see text), it is not possible to recover absolute risk adjusted length of hospital stay levels directly.

*ICD-10 codes.

earlier analysis of the changes in London and Greater Manchester, which found that the centralisation in London, where most patients were treated in a HASU, resulted in reductions in mortality, whereas the 2010 Greater Manchester centralisation, in which most patients were not treated in a HASU, did not. This finding is strengthened by our new analysis of the further centralisation in Greater Manchester, in which most patients were treated in a HASU and which showed reductions in mortality. These findings are also supported by data presented here showing the increasing numbers of patients treated at a HASU in Greater Manchester and our analysis of the provision of evidence based clinical interventions (that is, that these were more likely to be achieved after the centralisation in London compared with the first centralisation in Greater Manchester, and that achievement significantly increased with the further centralisation in Greater Manchester and was largely maintained in London).

Our earlier findings on clinical outcomes in London and Greater Manchester were presented in the 2014 NHS Five Year Forward View as evidence of the

\begin{tabular}{|c|c|c|c|c|c|c|c|}
\hline \multirow[b]{2}{*}{ Intervention } & \multicolumn{4}{|c|}{ Greater Manchester: risk to adjusted proportions $(95 \% \mathrm{Cl})$} & \multicolumn{3}{|c|}{ Rest of England: risk to adjusted proportions $(95 \% \mathrm{Cl})$} \\
\hline & Ramsay* & $2013 / 14$ & $2014 / 15$ & $2015 / 16$ & $2013 / 14$ & $2014 / 15$ & $2015 / 16$ \\
\hline $\begin{array}{l}\text { Brain scan within } \\
60 \text { min }\end{array}$ & & 40.7 (39.1 to 42.2$)$ & $49.2(47.6$ to 50.8$)$ & $60.8(59.4$ to 62.2$)$ & $43.0(42.6$ to 43.4$)$ & 44.8 (44.4 to 45.2) & $47.6(47.3$ to 48.0$)$ \\
\hline $\begin{array}{l}\text { Brain scan within } \\
180 \mathrm{~min}\end{array}$ & $65.2(64.3$ to 66.2$)$ & $74.1(72.7$ to 75.4$)$ & 81.7 (80.5 to 82.9 ) & $86.4(85.4$ to 87.3$)$ & 70.9 (70.6 to 71.3$)$ & $74.0(73.7$ to 74.3$)$ & $77.0(76.7$ to 77.3$)$ \\
\hline Brain scan within $24 \mathrm{~h}$ & $94.0(93.5$ to 94.4$)$ & 95.1 (94.4 to 95.8) & $97.1(96.5$ to 97.6$)$ & 97.4 (96.9 to 97.9) & 94.9 (94.8 to 95.1) & $96.0(95.8$ to 96.1$)$ & 96.7 (96.6 to 96.8$)$ \\
\hline tPA to eligible patients & & 67.9 (63.6 to 72.1$)$ & $82.3(78.1$ to 86.5$)$ & 88.6 (85.5 to 91.7) & 80.6 (79.8 to 81.3$)$ & 89.1 (88.5 to 89.7) & 91.8 (91.3 to 92.4) \\
\hline tPA within $60 \mathrm{~min}$ & & 74.4 (69.6 to 79.2$)$ & 70.5 (65.1 to 75.8$)$ & $74.6(70.2$ to 78.9$)$ & 53.1 (52.0 to 54.2) & 57.1 (56.0 to 58.1$)$ & 59.7 (58.6 to 60.7$)$ \\
\hline $\begin{array}{l}\text { Swallow screen within } \\
4 \mathrm{~h}\end{array}$ & & 58.7 (57.1 to 60.3) & $63.4(61.8$ to 65.0$)$ & 69.2 (67.8 to 70.6$)$ & 59.7 (59.3 to 60.1) & 63.9 (63.5 to 64.2) & 67.7 (67.3 to 68.0) \\
\hline $\begin{array}{l}\text { Admitted to SU within } \\
4 \mathrm{~h}\end{array}$ & $55.9(54.9$ to 57.0$)$ & 57.4 (55.8 to 59.1$)$ & $58.8(57.1$ to 60.5$)$ & 79.1 (77.9 to 80.4$)$ & 51.3 (50.9 to 51.7$)$ & 52.8 (52.4 to 53.2$)$ & 53.4 (53.0 to 53.7$)$ \\
\hline $\begin{array}{l}\text { Consultant assessment } \\
14 \mathrm{~h}\end{array}$ & & 57.6 (55.9 to 59.2$)$ & $58.9(57.2$ to 60.6$)$ & 79.2 (78.0 to 80.4$)$ & $50.6(50.2$ to 51.0$)$ & 52.1 (51.7 to 52.5$)$ & $52.6(52.2$ to 53.0$)$ \\
\hline $\begin{array}{l}\text { Consultant assessment } \\
24 \mathrm{~h}\end{array}$ & & 78.2 (76.9 to 79.6$)$ & 80.2 (78.9 to 81.5$)$ & 93.0 (92.3 to 93.8) & 81.1 (80.8 to 81.4$)$ & $82.8(82.5$ to 83.1$)$ & $84.3(84.1$ to 84.6$)$ \\
\hline $\begin{array}{l}\text { Stroke nurse } \\
\text { assessment } 12 \mathrm{~h}\end{array}$ & & $86.4(85.3$ to 87.4$)$ & 86.6 (85.5 to 87.7$)$ & 91.2 (90.4 to 92.0) & 86.5 (86.2 to 86.7$)$ & 88.1 (87.8 to 88.3 ) & 89.3 (89.0 to 89.5$)$ \\
\hline $\begin{array}{l}\text { Stroke nurse } \\
\text { assessment } 24 \mathrm{~h}\end{array}$ & & 93.4 (92.7 to 94.2 ) & 92.8 (92.0 to 93.6) & 94.6 (93.9 to 95.2) & 93.2 (93.0 to 93.4) & 93.9 (93.7 to 94.0 ) & 94.5 (94.4 to 94.7$)$ \\
\hline $\begin{array}{l}\text { Physiotherapist } \\
\text { assessment } 24 \mathrm{~h}\end{array}$ & & $55.4(53.7$ to 57.0$)$ & $63.2(61.6$ to 64.8$)$ & 80.7 (79.5 to 81.9) & $55.6(55.2$ to 56.0$)$ & 57.1 (56.7 to 57.4$)$ & 59.7 (59.3 to 60.1$)$ \\
\hline $\begin{array}{l}\text { Physiotherapist } \\
\text { assessment } 72 \mathrm{~h}\end{array}$ & 92.1 (91.5 to 92.7) & 95.1 (94.3 to 95.8) & 97.0 (96.4 to 97.6) & 97.5 (97.1 to 98.0 ) & 93.6 (93.4 to 93.8) & 93.4 (93.2 to 93.6) & 93.7 (93.5 to 93.9) \\
\hline $\begin{array}{l}\text { Occupational therapist } \\
24 \mathrm{~h}\end{array}$ & & 50.5 (48.8 to 52.2$)$ & 61.2 (59.6 to 62.8$)$ & 79.4 (78.1 to 80.6$)$ & $45.2(44.7$ to 45.6$)$ & $47.5(47.1$ to 47.9$)$ & 52.3 (51.9 to 52.7$)$ \\
\hline $\begin{array}{l}\text { Occupational therapist } \\
72 \mathrm{~h}\end{array}$ & & 94.4 (93.6 to 95.1) & 96.4 (95.8 to 97.0) & 96.9 (96.4 to 97.4$)$ & $87.3(87.1$ to 87.6$)$ & 88.5 (88.2 to 88.7$)$ & 89.7 (89.4 to 89.9 ) \\
\hline SaLT swallow $24 \mathrm{~h}$ & & $53.2(50.8$ to 55.6$)$ & $56.9(54.4$ to 59.4$)$ & 55.7 (53.1 to 58.2) & $48.4(47.8$ to 49.0$)$ & 49.9 (49.3 to 50.5) & $54.0(53.4$ to 54.6$)$ \\
\hline SaLT swallow $72 \mathrm{~h}$ & 94.0 (93.6 to 94.4) & 85.5 (83.9 to 87.2$)$ & 91.3 (89.9 to 92.7) & 91.9 (90.6 to 93.3) & 78.8 (78.3 to 79.3$)$ & 82.2 (81.7 to 82.6) & $84.9(84.5$ to 85.3$)$ \\
\hline $\begin{array}{l}\text { SaLT communication } \\
24 \mathrm{~h}\end{array}$ & & 39.7 (37.3 to 42.2 ) & 53.8 (51.5 to 56.2$)$ & 69.9 (68.0 to 71.7) & 35.5 (34.9 to 36.1$)$ & $38.0(37.5$ to 38.6$)$ & $42.0(41.5$ to 42.6$)$ \\
\hline $\begin{array}{l}\text { SaLT communication } \\
72 \mathrm{~h}\end{array}$ & & 86.7 (85.1 to 88.3 ) & 93.4 (92.2 to 94.5) & 96.6 (95.9 to 97.3) & 78.3 (77.8 to 78.7$)$ & $81.6(81.2$ to 82.0$)$ & 85.1 (84.7 to 85.5$)$ \\
\hline
\end{tabular}

$\mathrm{SaLT}=$ speech and language therapist; $\mathrm{SU}=$ stroke unit; $\mathrm{tPA}=$ tissue plasminogen activator.

* Refers to data from period 2010-12. ${ }^{20}$

See supplementary material for graphical depiction. 


\begin{tabular}{|c|c|c|c|c|}
\hline Intervention & Ramsay* & $2013 / 14$ & $2014 / 15$ & $2015 / 16$ \\
\hline Brain scan within $60 \mathrm{~min}$ & & $57.3(56.2$ to 58.3$)$ & 58.9 (57.9 to 60.0$)$ & 59.9 (58.8 to 61.0$)$ \\
\hline Brain scan within $180 \mathrm{~min}$ & 66.3 (65.6 to 67.1$)$ & 80.0 (79.1 to 80.9$)$ & 81.8 (81.0 to 82.6$)$ & $82.2(81.4$ to 83.1$)$ \\
\hline Brain scan within $24 \mathrm{~h}$ & $95.2(94.8$ to 95.5$)$ & 97.2 (96.9 to 97.6$)$ & 97.1 (96.8 to 97.5$)$ & 97.3 (97.0 to 97.7$)$ \\
\hline tPA to eligible patients & & 92.9 (91.6 to 94.2$)$ & $94.4(93.2$ to 95.7$)$ & 88.6 (86.9 to 90.3$)$ \\
\hline tPA within $60 \mathrm{~min}$ & & 83.0 (81.0 to 85.0$)$ & 83.9 (81.9 to 85.9$)$ & 80.8 (78.6 to 83.1$)$ \\
\hline Swallow screen within $4 \mathrm{~h}$ & & 65.1 (64.1 to 66.2$)$ & 68.1 (67.1 to 69.2$)$ & 70.6 (69.6 to 71.6$)$ \\
\hline Admitted to SU within $4 \mathrm{~h}$ & 66.3 (65.6 to 67.1$)$ & 61.1 (60.0 to 62.1) & 59.8 (58.7 to 60.8$)$ & $60.3(59.2$ to 61.3$)$ \\
\hline Consultant assessment $14 \mathrm{~h}$ & & $50.3(49.2$ to 51.5$)$ & 53.7 (52.6 to 54.8$)$ & $51.3(50.1$ to 52.4$)$ \\
\hline Consultant assessment $24 \mathrm{~h}$ & & $89.3(88.6$ to 90.0$)$ & 90.2 (89.6 to 90.9) & $88.5(87.8$ to 89.2$)$ \\
\hline Stroke nurse assessment $12 \mathrm{~h}$ & & $90.8(90.1$ to 91.4$)$ & 92.7 (92.1 to 93.2) & $93.3(92.7$ to 93.8$)$ \\
\hline Stroke nurse assessment $24 \mathrm{~h}$ & & $95.5(95.1$ to 96.0$)$ & $95.6(95.2$ to 96.1$)$ & $96.2(95.7$ to 96.6$)$ \\
\hline Physiotherapist assessment $24 \mathrm{~h}$ & & $57.4(56.2$ to 58.5$)$ & 61.7 (60.5 to 62.8) & $64.7(63.6$ to 65.8$)$ \\
\hline Physiotherapist assessment $72 \mathrm{~h}$ & 95.4 (95.0 to 95.8) & $95.0(94.5$ to 95.5$)$ & 96.5 (96.0 to 96.9) & $95.8(95.3$ to 96.2$)$ \\
\hline Occupational therapist $24 \mathrm{~h}$ & & $50.3(49.1$ to 51.4$)$ & $57.0(55.8$ to 58.1$)$ & $61.5(60.3$ to 62.6$)$ \\
\hline Occupational therapist $72 \mathrm{~h}$ & & 91.1 (90.5 to 91.8) & $94.6(94.1$ to 95.1$)$ & 94.1 (93.6 to 94.7$)$ \\
\hline SaLT swallow $24 \mathrm{~h}$ & & $53.9(52.2$ to 55.7$)$ & 54.1 (52.5 to 55.8) & $51.5(49.8$ to 53.3$)$ \\
\hline SaLT swallow $72 \mathrm{~h}$ & 98.2 (97.9 to 98.4) & 89.5 (88.5 to 90.6$)$ & 91.8 (90.9 to 92.7) & $88.4(87.3$ to 89.5$)$ \\
\hline SaLT communication $24 \mathrm{~h}$ & & $46.4(45.0$ to 47.8$)$ & 50.1 (48.7 to 51.5$)$ & $50.7(49.2$ to 52.1$)$ \\
\hline SaLT communication $72 \mathrm{~h}$ & & 89.7 (88.8 to 90.5$)$ & $93.0(92.3$ to 93.7$)$ & $91.0(90.2$ to 91.8$)$ \\
\hline
\end{tabular}

“case for greater concentration of care," associated guidance on transforming urgent and emergency care services across the English NHS, and the National Clinical Strategy for Scotland 2016. ${ }^{19}$ 39-41 Our findings on clinical interventions have been cited in the 2016 edition of the National Clinical Guidance for Stroke as evidence of the benefits of stroke service reorganisation..$^{20}$ On the basis of the experiences in London and Greater Manchester, several areas of the UK (including both urban and more rural areas) are considering system-wide reconfiguration of acute stroke services and have cited our earlier research in "case for change" documents; these areas include Yorkshire and the Humber; Birmingham, Solihull, and the Black Country; Sussex; Kent and Medway; South West England; and Cumbria. ${ }^{43-49}$ Our methods and findings may also inform the centralisation of other healthcare services such as cancer care, ${ }^{50}{ }^{51}$ cardiac arrest/myocardial infarction, ${ }^{52-54}$ major trauma care, ${ }^{55}$ and vascular surgery, ${ }^{56}$ for which evidence of impact is growing.

\section{Unanswered questions and future research}

Given that we could analyse data only for patients treated up to one year after the second centralisation in Greater Manchester, and the fact that SSNAP data indicate that the proportion of patients treated in a HASU and receiving clinical interventions has continued to increase in Greater Manchester, ${ }^{32}$ further research on the longer term effects of the second reconfiguration in Greater Manchester would be beneficial. Future research would also be beneficial to examine effects on disability and dependence after stroke, as well as how and why the improvements seen in London were sustained over time. Given the results of this and our previous study, further research would be helpful to understand how to further improve access to HASU care for all stroke patients. In April 2017 NHS
England announced that it will begin commissioning mechanical thrombectomy for patients with certain types of acute ischaemic stroke. ${ }^{57}$ Further work to evaluate the effect of introducing these services into the NHS, and how they ought to be organised, would be useful.

\section{AUTHOR AFFILIATIONS}

${ }^{1}$ Department of Applied Health Research, University College London, London WC1E 7HB, UK

${ }^{2}$ Alliance Manchester Business School, University of Manchester, Manchester M15 6PB, UK

${ }^{3}$ Research Department of Primary Care and Population Health, University College London, London NW3 2PF, UK

${ }^{4}$ Department of Population Health Sciences, School of Population Heath and Environmental Sciences, King's College London, London SE1 1UL, UK

${ }^{5}$ Stroke Programme, Royal College of Physicians, London, UK

${ }^{6}$ Guy's and St Thomas' NHS Foundation Trust, St Thomas' Hospital, London SE1 7EH, UK

${ }^{7}$ Health Policy, Politics and Organisation (HiPPO) Research Group, Centre for Primary Care, School of Health Sciences, University of Manchester, Manchester M13 9PL, UK

${ }^{8}$ Stroke and Vascular Centre, University of Manchester, Manchester Academic Health Science Centre, Salford Royal Hospitals NHS Foundation Trust, Salford M6 8HD, UK

${ }^{9}$ National Institute of Health Research Comprehensive Biomedical Research Centre, Guy's and St Thomas' NHS Foundation Trust and King's College London, Guy's Hospital, London SE1 9RT, UK

We thank the many people and organisations participating in the Sentinel Stroke National Audit Programme (SSNAP), as well as members of the SSNAP collaboration (www.strokeaudit.org/Research/ SSNAP-Collaboration.aspx)

Contributors: SM, NJF, and AIGR designed the study. SM did the analysis of the Hospital Episodes Statistics data and drafted the manuscript. AIGR did the analysis of the SSNAP data. SM, AIGR, RB, RMH, CM, LP, CP, AGR, SJT, PJT, CDAW, and NJF made substantial contributions to the study design and interpretation of the findings. All authors contributed to the drafting of the article or critical revision for important intellectual content, gave final approval of the version to be published, and agree to be accountable for all aspects of the work in ensuring that questions related to the accuracy or integrity of any part of the article are appropriately investigated and resolved. The corresponding author attests that all listed authors meet authorship 
criteria and that no others meeting the criteria have been omitted. SM and NJF are the guarantors.

Funding: This paper presents independent research commissioned by the National Institute for Health Research (NIHR) Health Services and Delivery Research Programme, funded by the Department of Health (study reference 10/1009/09). The views expressed are those of the authors and not necessarily those of the NHS, the NIHR, or the Department of Health and Social Care. SM and NJF were supported by the NIHR Collaboration for Leadership in Applied Health Research and Care (CLAHRC) North Thames at Barts Health NHS Trust. CW and CM were supported by the NIHR Biomedical Research Centre at Guy's and St Thomas' National Health Service Foundation Trust and King's College London, and the south London NIHR CLAHRC. RB was supported by the NIHR CLAHRC Greater Manchester. SSNAP is commissioned by the Healthcare Quality Improvement Partnership and funded by NHS England and the Welsh Government. The sponsor approved all aspects of the study protocol and any amendments thereto, but played no other role in design or conduct of the study.

Competing interests: All authors have completed the ICMJE uniform disclosure form at http://www.icmje.org/coi_disclosure.pdf (available on request from the corresponding author) and declare: no support from any organisation for the submitted work other than that described above; no financial relationships with any organisations that might have an interest in the submitted work in the previous three years; AGR is national clinical director for stroke in England and clinical director for stroke in London; PJT was clinical lead for stroke in Greater Manchester up to 2013 and led the Greater Manchester stroke service redesign from 2007 until 2012.

Ethical approval: The study received ethical approval in September 2011 from the London East NHS Research Ethics Committee (ref ${ }^{11} /$ LO/1396).

Data sharing: No additional data available.

Transparency: The lead author affirms that this manuscript is an honest, accurate, and transparent account of the study being reported; that no important aspects of the study have been omitted; and that any discrepancies from the study as planned (and, if relevant, registered) have been explained.

This is an Open Access article distributed in accordance with the terms of the Creative Commons Attribution (CC BY 4.0) license, which permits others to distribute, remix, adapt and build upon this work, for commercial use, provided the original work is properly cited. See: http://creativecommons.org/licenses/by/4.0/.

1 Feigin VL, Roth GA, Naghavi M, et al, Global Burden of Diseases, Injuries and Risk Factors Study 2013 and Stroke Experts Writing Group. Global burden of stroke and risk factors in 188 countries, during 1990-2013: a systematic analysis for the Global Burden of Disease Study 2013. Lancet Neurol 2016;15:913-24. doi:10.1016/ S1474-4422(16)30073-4

2 Bray BD, Ayis S, Campbell J, et al. Associations between the organisation of stroke services, process of care, and mortality in England: prospective cohort study. BMJ 2013;346:f2827. doi:10.1136/bmj.f2827

3 Langhorne P, Fearon P, Ronning OM, et al, Stroke Unit Trialists' Collaboration. Stroke unit care benefits patients with intracerebral hemorrhage: systematic review and meta-analysis. Stroke 2013;44:3044-9. doi:10.1161/ STROKEAHA.113.001564

4 Stroke Unit Trialists' Collaboration. Organised inpatient (stroke unit) care for stroke. Cochrane Database Syst Rev 2013:11:CD000197.

5 Aguiar de Sousa D, von Martial R, Abilleira S, et al. Access to and delivery of acute ischaemic stroke treatments: A survey of national scientific societies and stroke experts in 44 European countries. Eur Stroke / (forthcoming).

6 Wiedmann S, Hillmann S, Abilleira S, et al, European Implementation Score Collaboration. Variations in acute hospital stroke care and factors influencing adherence to quality indicators in 6 European audits. Stroke 2015;46:579-81. doi:10.1161/ STROKEAHA.114.007504

7 Arnao V, Popovic N, Caso V. How is stroke care organised in Europe? Presse Med 2016:45:e399-408 doi:10.1016/i.lpm.2016.10.004

8 Higashida R, Alberts MJ, Alexander DN, et al, American Heart Association Advocacy Coordinating Committee. Interactions within stroke systems of care: a policy statement from the American Heart Association/American Stroke Association. Stroke 2013;44:2961-84. doi:10.1161/STR.0b013e3182a6d2b2

9 Prabhakaran S, O'Neill K, Stein-Spencer L, Walter J, Alberts MJ. Prehospital triage to primary stroke centers and rate of stroke thrombolysis. JAMA Neurol 2013;70:1126-32. doi:10.1001/ jamaneurol.2013.293
10 Weir NU, Buchan AM. A study of the workload and effectiveness of a comprehensive acute stroke service. I Neurol Neurosurg Psychiatry 2005;76:863-5. doi:10.1136/jnnp.2004.053462

11 Swartz RH, Linkewich E, Sharp S, et al. A system-based intervention to improve access to hyperacute stroke care. Can J Neurol Sci 2017:44:475-82. doi:10.1017/cjn.2017.40

12 Lahr MM, Luijckx G-J, Vroomen PC, van der Zee DJ, Buskens E. Proportion of patients treated with thrombolysis in a centralized versus a decentralized acute stroke care setting. Stroke 2012;43:1336-40. doi:10.1161/STROKEAHA.111.641795

13 Douw K, Nielsen CP, Pedersen CR. Centralising acute stroke care and moving care to the community in a Danish health region: Challenges in implementing a stroke care reform. Health Policy 2015;119:1005 10. doi:10.1016/j.healthpol.2015.05.007

14 Cadilhac DA, Purvis T, Kilkenny MF, et al, New South Wales Strokes Services Coordinating Committee, Agency for Clinical Innovation. Evaluation of rural stroke services: does implementation of coordinators and pathways improve care in rural hospitals? Stroke 2013;44:2848-53. doi:10.1161/STROKEAHA.113.001258

15 Lahr MM, Luijckx G-J, Vroomen PC, van der Zee DJ, Buskens E. The chain of care enabling tPA treatment in acute ischemic stroke: a comprehensive review of organisational models. $J$ Neurol 2013;260:960-8 doi:10.1007/s00415-012-6647-7

16 Langhorne P, Lewsey JD, Jhund PS, et al. Estimating the impact of stroke unit care in a whole population: an epidemiological study using routine data. / Neurol Neurosurg Psychiatry 2010;81:1301-5. doi:10.1136/jnnp.2009.195131

17 Hastrup S, Johnsen SP, Terkelsen T, et al. Effects of centralizing acute stroke services: A prospective cohort study. Neurology 2018;91:e236-48. doi:10.1212/ WNL.0000000000005822

18 Fulop N, Boaden R, Hunter R, et al. Innovations in major system reconfiguration in England: a study of the effectiveness, acceptability and processes of implementation of two models of stroke care. Implement Sci 2013;8:5. doi:10.1186/1748-5908-8-5

19 Morris S, Hunter RM, Ramsay Al, et al. Impact of centralising acute stroke services in English metropolitan areas on mortality and length of hospital stay: difference-in-differences analysis. BMJ 2014;349:g4757. doi:10.1136/bmj.g4757

20 Ramsay Al, Morris S, Hoffman A, et al. Effects of centralizing acute stroke services on stroke care provision in two large metropolitan areas in England. Stroke 2015;46:2244-51. doi:10.1161/ STROKEAHA.115.009723

21 Hunter RM, Fulop NJ, Boaden RJ, et al. The potential role of cost-utility analysis in the decision to implement major system change in acute stroke services in metropolitan areas in England. Health Res Policy Syst 2018;16:23. doi:10.1186/s12961-018-0301-5

22 Friebel R, Hauck K, Aylin P. Centralisation of acute stroke services in London: Impact evaluation using two treatment groups. Health Econ 2018;27:722-32. doi:10.1002/hec.3630

23 Balinskaite V, Bottle A, Shaw LJ, Majeed A, Aylin P. Reorganisation of stroke care and impact on mortality in patients admitted during weekends: a national descriptive study based on administrative data. BMJ Qual Saf 2018;27:611-8. doi:10.1136/bmjqs-2017-006681

24 Hunter RM, Davie C, Rudd A, et al. Impact on clinical and cost outcomes of a centralized approach to acute stroke care in London: a comparative effectiveness before and after model. PLOS One 2013;8:e70420. doi:10.1371/journal.pone.0070420

25 Imison C, Sonola L, Honeyman M, Ross S. The reconfiguration of clinical services in the NHS: what is the evidence? King's Fund, 2014

26 Greater Manchester and Cheshire Cardiac and Stroke Network Support Team. Development of stroke services in Greater Manchester: twelve month review. Greater Manchester and Cheshire Cardiac and Stroke Network, 2011.

27 NHS Digital. Hospital Episode Statistics. 2018. https://digital.nhs. uk/data-and-information/data-tools-and-services/data-services/ hospital-episode-statistics.

28 Intercollegiate Stroke Working Party. National clinical guideline for stroke. 4th ed. Royal College of Physicians, 2012.

29 Office for National Statistics. 2001 rural-urban classification. 2018. https://www.ons.gov.uk/ methodology/geography/geographicalproducts/ ruralurbanclassifications/2001 ruralurbanclassification.

30 NHS Digital. Linked HES-ONS mortality data. 2018. https://digital. nhs.uk/data-and-information/data-tools-and-services/data-services/ linked-hes-ons-mortality-data.

31 Department of Health. National stroke strategy. Department of Health, 2007

32 Royal College of Physicians Clinical Effectiveness and Evaluation Unit. Sentinel Stroke National Audit Programme (SSNAP) clinical audit public report (December 2016 - March 2017). Royal College of Physicians, 2017.

33 Data.gov.uk. Index of Multiple Deprivation 2004. 2014 https://data. gov.uk/dataset/59599787-bd50-4500-a409-fc586260dbbd/ index-of-multiple-deprivation-2004. 
34 Sutton M, Nikolova S, Boaden R, Lester H, McDonald R, Roland M. Reduced mortality with hospital pay for performance in England. N Engl/ Med 2012;367:1821-8. doi:10.1056/NEIMsa1114951

35 Barber J, Thompson S. Multiple regression of cost data: use of generalised linear models. J Health Serv Res Policy 2004;9:197-204. doi:10.1258/1355819042250249

36 Royal College of Physicians Clinical Effectiveness and Evaluation Unit. SSNAP - Clinical audit August-November 2016 public report. Royal College of Physicians, 2017.

37 Pickering A, Harnan S, Cooper K, Sutton A, Mason S, Nicholl J. Acute ischaemic stroke patients - direct admission to a specialist centre or initial treatment in a local hospital? A systematic review. J Health Serv Res Policy 2016;21:51-60. doi:10.1177/1355819615586006

38 Hubert GJ, Meretoja A, Audebert HJ, et al. Stroke thrombolysis in a centralized and a decentralized system (Helsinki and telemedical project for integrative stroke care network). Stroke 2016;47:29993004. doi:10.1161/STROKEAHA.116.014258

39 NHS England, Public Health England, Health Education England Monitor, Care Quality Commission, NHS Trust Development Authority. Five year forward view. NHS England, 2014.

40 Urgent and Emergency Care Review Team and Emergency Care Intensive Support Team. Safer, faster, better: good practice in delivering urgent and emergency care - a guide for local health and social care communities. NHS England, 2015.

41 Scottish Government. A national clinical strategy for Scotland. Scottish Government, 2016

42 Intercollegiate Stroke Working Party. National clinical guideline for stroke. 5th ed. Royal College of Physicians, 2016.

43 Brown R. Stroke - End of phase 1 report - Case for change. 2015 https://smybndccgs.nhs.uk/application/files/1014/8300/8792/ Case_for_change.pdf.

44 Yorkshire and the Humber Strategic Clinical Network. Hyper acute stroke services: Yorkshire and the Humber 'Blueprint' for Yorkshire and the Humber Clinical Commissioning Group. 2016. https:// smybndccgs.nhs.uk/application/files/8014/8300/8726/Yorkshire and_Humber_blueprint_for_HASS_for_CCGs.pdf.

45 West Midlands Clinical Senate. Stroke service reconfiguration review for Birmingham, Solihull and the Black Country. 2015. http://www. wmscnsenate.nhs.uk/files/8814/4525/8647/WMCS_Stroke_ Service_Reconfiguration_Review_for_Birmingham_Solihull_and_ the_Black_Country_Final_Report_v10ct2015.pdf

46 South East Clinical Senate. Review of proposals for future stroke services in Sussex. 2015. http://www.secsenate.nhs. uk/files/7514/5321/3862/South_East_Clinical_Senate_ Review of proposals for future stroke services in Sussex Dec_2015.pdf.
47 Kent \& Medway Public Health Observatory. Kent and Medway stroke services: evidence review of hyperacute stroke units. 2015. https:// www.kpho.org.uk/_data/assets/pdf_file/0009/55566/Strokeevidence-final-version-for-submission.pdf.

48 South West Cardiovascular Strategic Clinical Network. Bigger, better, faster? An options appraisal for the reconfiguration of emergency heart attack and stroke services for the South West of England. 2016 http://www.swscn.org.uk/wp/wp-content/uploads/2013/12/SW-CV SCN-Bigger-Better-Faster-Full-Report-September-2016.pdf.

49 NHS North Cumbria CCG. Implementation Reference Group report: community hospitals and acute stroke care. 2018. http://www.northcumbriaccg.nhs.uk/about-us/how-we-makedecisions/Governing-Body-Meetings/2018/2018-april/ bulk/07implementationreferencegrouprecommendations.pdf.

50 Nelen SD, Heuthorst L, Verhoeven RHA, et al. Impact of centralizing gastric cancer surgery on treatment, morbidity, and mortality. I Gastrointest Surg 2017;21:2000-8. doi:10.1007/s11605-017-3531-x

51 Wouters MW, Karim-Kos HE, le Cessie S. Centralization of esophageal cancer surgery: does it improve clinical outcome? Ann Surg Oncol 2009:16:1789-98, doi:10.1245/s10434-009-0458-9

52 Fothergill RT, Watson LR, Virdi GK, Moore FP, Whitbread M, et al. Survival of resuscitated cardiac arrest patients with ST-elevation myocardial infarction (STEMI) conveyed directly to a Heart Attack Centre by ambulance clinicians. Resuscitation 2014;85:96-8. doi:10.1016/j.resuscitation.2013.09.010

53 Miles S. Regionalised cardiac arrest centres as a means to improve outcomes from out-of-hospital cardiac arrest in the UK: a literature review. Br Paramed / 2016;1:23810.29045/14784726.2016.1.3.23.

54 Schober A, Sterz F, Laggner AN, et al. Admission of out-of-hospital cardiac arrest victims to a high volume cardiac arrest center is linked to improved outcome. Resuscitation 2016;106:42-8. doi:10.1016/j. resuscitation.2016.06.021

55 Pickering A, Cooper K, Harnan S, Sutton A, Mason S, Nicholl J. Impact of prehospital transfer strategies in major trauma and head injury: systematic review, meta-analysis, and recommendations for study design. J Trauma Acute Care Surg 2015;78:164-77. doi:10.1097/ TA.0000000000000483

56 Partridge E, Brooks M, Curd C, Davis V, Oates C, McGeeney D. The effects of centralisation of vascular surgical services in the Bath, Bristol and Weston area on the carotid endarterectomy pathway. Ann R Coll Surg Engl 2017;99:617-23. doi:10.1308/rcsann.2017.0087

57 NHS England. Stroke patients in England set to receive revolutionary new treatment. 2017. https://www.england.nhs.uk/2017/04/strokepatients-in-england-set-to-receive-revolutionary-new-treatment/.

Web appendix: Supplementary material 